\title{
Tanshinone production could be increased by the expression of SmWRKY2 in Salvia miltiorrhiza hairy roots
}

\author{
Changping Deng ${ }^{\mathrm{a}, \mathrm{b}, 1}$, Xiaolong Hao ${ }^{\mathrm{b}, 1}$, Min Shi ${ }^{\mathrm{a}}$, Rong Fu ${ }^{\mathrm{b}}$, Yao Wang ${ }^{\mathrm{a}}$, Yi Zhang ${ }^{\mathrm{b}}$, Wei Zhou ${ }^{\mathrm{a}}$, \\ Yue Feng ${ }^{\mathrm{a}}$, Nokwanda P. Makunga ${ }^{\mathrm{c}, *}$, Guoyin $\mathrm{Kai}^{\mathrm{a}}{ }^{\mathrm{a}, *}$ \\ a Laboratory of Medicinal Plant Biotechnology, College of pharmacy, Zhejiang Chinese Medical University, Hangzhou, Zhejiang, 310053, PR China \\ ${ }^{\mathrm{b}}$ Institute of Plant Biotechnology, School of Life Sciences, Shanghai Normal University, Shanghai, 200234, PR China \\ ${ }^{\mathrm{c}}$ Department of Botany and Zoology, Stellenbosch University, Private Bag X1, Matieland, 7600, South Africa
}

\section{A R T I C L E I N F O}

\section{Keywords:}

Hairy roots

S.miltiorrhiza

Tanshinones

WRKY transcription factor

\begin{abstract}
A B S T R A C T
Tanshinones are the main bioactive diterpenes in Salvia miltiorrhiza Bunge, are widely used for treating cardiovascular and cerebrovascular diseases. However, the biosynthetic mechanisms of these compounds have not yet been fully explained. In this study, a transcription factor named SmWRKY2 was isolated and functionally characterized. Multiple sequence analysis indicated it was classified into subgroup I of the WRKY family. Expression pattern showed that SmWRKY2 was mainly expressed in the stem and leaf and was inducible by methyl jasmonate (MeJA) treatment. Subcellular localization showed that SmWRKY2 was localized in the nucleus. Overexpression of SmWRKY2 in S. miltiorrhiza hairy roots significantly increased the expression of $S m D X S 2$ and SmCPS, resulting in increased accumulation of tanshinones and the highest total tanshinone content was detected in OE-SmWRKY2-1 line, which was 1.83 times of the control. Meanwhile, tanshinone production was slightly reduced in the antisense-SmWRKY2 line. Dual-Luciferase assay showed that SmWRKY2 can positively regulate SmDXS2 and SmCPS expression, However, Y1H and EMSA experiments indicate that SmWRKY2 only binds to the W-box of the SmCPS promoter. Our study shows that SmWRKY2 is a positive regulator of tanshinone biosynthesis by mainly activating SmCPS. This study thus sheds new light on the regulatory role of SmWRKY2 in tanshinone biosynthesis.
\end{abstract}

\section{Introduction}

Cardiovascular and cerebrovascular diseases are one of the most serious health problems, threatening people's lives all around the word [1]. S. miltiorrhiza (also named Danshen), a well-known and valuable traditional Chinese medicine, has been proven to be effective in the treatment of cardiovascular diseases, and this plant has also been developed into various preparations such as the famous Compound Danshen Dripping Pills, which are now widely used in many countries [2-5]. There are mainly two categories of bioactive compounds in Danshen, namely tanshinones and phenolic acids $[3,4,6,7]$. As one kind of diterpenoids, tanshinones including dihydrotanshinone (DH-TI), tanshinone IIA (T-IIA), cryptotanshinone (CT) and tanshinone I (T-I) are derived from the universal five-carbon precursor isopentenyl pyrophosphate (IPP) and its isomer dimethylallyl pyrophosphate (DMAPP) that is synthesized in two independent pathways, the 2-C-methyl-Derythritol 4-phosphate (MEP) pathway that has a plastidial location and the mevalonate (MVA) pathway in the cytosol (Fig. S1) [3,8-11]. Recently, multiple rate-limiting genes involved in tanshinone biosynthesis have been isolated and identified from $S$. miltiorrhiza, such as those encoding for 3-hydroxy-3-methylglutaryl CoA reductase (HMGR), 1Deoxy-D-xylulose 5-phosphate synthase (DXS2),1-deoxy D-5-phosphate reductoisomerase (DXR), geranylgeranyl diphosphate synthase (GGPPS), copalyl diphosphate synthase (CPS), kaurene synthase-like (KSL), miltiradiene oxidase (CYP76AH1), to name a few [3,6,12-14]. Manipulating key enzyme genes by genetic engineering individually or

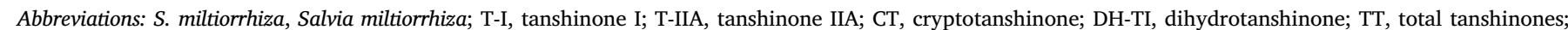

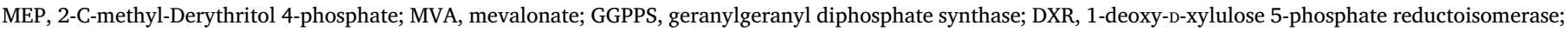

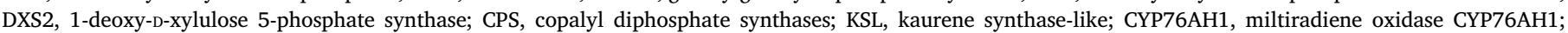
HMGR, 3-hydroxy-3-methylglutaryl CoA reductase; MJ, methyl jasmonate; pI, isoelectric points; TF, transcription factor

* Corresponding authors.

E-mail addresses: makunga@sun.ac.za (N.P. Makunga), kaiguoyin@zcmu.edu.cn (G. Kai).

${ }^{1}$ Co-first authors. 
in-combination has been tested as an alternative method for higher production of tanshinones. For example, the introduction of SmHMGR, SmDXS2, SmGGPPS or co-expression of SmHMGR-SmGGPPS, SmHMGRSmDXR and SmGGPPS-SmDXS2 could elevate concentrations of tanshinones in $S$. miltiorrhiza hairy roots $[3,4,15]$. Nevertheless, complete downstream committed steps in tanshinone biosynthesis remain unclear, which narrows the application of genetic engineering.

Studies have shown that it is an effective approach to enhance the secondary metabolites content by manipulating transcription factors in plants [16]. With genome and transcriptome sequencing, multiple transcription factors (TF) have been identified in S. miltiorrhiza including bHLH, MYB, AP2/ERF and the WRKY family [17-20], and the function of several transcription factors have been validated. For example, overexpression of SmMYC2 could increase the production of phenolic acids in transgenic $S$. miltiorrhiza seedlings [5]. Introduction of the SmERF1L1 transcription factor positively regulated tanshinone accumulation in Danshen hairy roots [11], while SmERF115 played an important role in phenolic acid production [21]. A R2R3-MYB gene $S m M Y B 9 b$ has been reported to improve tanshinone production in hairy roots [22], whereas relatively few studies have reported on the regulatory function of WRKY transcription factors in Danshen.

As one of the largest transcription factor families involved in plant kingdom, WRKY transcription factors play a broad role in plants $[20,23]$. WRKY transcription factors appear to have a common feature which is the WRKY domain that consists of 60 amino acids, with highly conserved WRKYGQK motif located in the N-terminus, and, a non-typical zinc-finger-like motif composed of $\mathrm{C} 2 \mathrm{HC}\left(\mathrm{C}-\mathrm{X}_{7}-\mathrm{C}-\mathrm{X}_{23}-\mathrm{H}-\mathrm{X}_{1}-\mathrm{C}\right)$ or $\mathrm{C} 2 \mathrm{H} 2\left(\mathrm{C}-\mathrm{X}_{4^{-}{ }^{-}}-\mathrm{C}-\mathrm{X}_{22^{-} 23^{2}}-\mathrm{H}-\mathrm{X}_{1}-\mathrm{H}\right)$ at the C-terminal [20,23-25]. According to the number of WRKY domains and zinc-finger-like motifs, WRKY transcription factors can thus be divided into three subfamilies $[20,23,24]$, and multiple studies have shown that the WRKY transcription factors can bind to the W-box (TTGACC/T) element, located in the promotor regions of the targeted genes [20,23,26-29]. Recently, dozens of studies focusing on WRKY transcription factors have shown their function in metabolism, signal processes and stress responses in many plant species, including traditional Chinese herbal plants [20,28-30]. For example, a group I WRKY from cotton named GhWRKY25 exhibited enhanced sensitivity to the fungal pathogen Botrytis cinereal in Nicotiana benthamiana [27]. A WRKY transcription factor GSW1, specifically expressed in the glandular trichomes of $\mathrm{Ar}$ temisia annua could enhance the levels of artemisinin and dihydroartemisinic acid through activating CYP71AV1 and AaORA expression as it binds to the W-box in promoter regions [29]. The Catharanthus roseus WRKY1 positively regulated the accumulation of terpenoid indole alkaloids (TIAs) in hairy root lines [30]. However, there are limited reports on the effects of WRKYs related to secondary metabolism in the model traditional herbal plant, $S$. miltiorrhiza and this presents with opportunities to further resolve the action of WRKYs in this important medicinal species.

In this study, we thus cloned and explored the function of MJ-inducible SmWRKY2 in $S$. miltiorrhiza. Multiple sequence analysis revealed that it contained two WRKY domains belonging to subgroup I of the WRKY family. Phylogenetic tree analysis showed that SmWRKY2 had high homology with AtWRKY4 that is found in Arabidopsis thaliana. We thus report on the overexpression of SmWRKY2 in S. miltiorrhiza, using hairy roots as system to explore the biosynthesis of tanshinones. This study also examined the effect of antisense-SmWRKY2 hairy root transgenesis on tanshinone metabolism. This present study reveals that SmWRKY2 may be a positive regulator participating in the biosynthesis of tanshinones by increasing the expression of SmDXS2 and SmCPS, which provides a new perspective for tanshinone biosynthesis.

\section{Materials and methods}

\subsection{Plant samples and reagents}

S. miltiorrhiza seedlings used for Agrobacterium-mediated transformation were cultivated in Murashige and Skoog (MS) medium (pH 5.8) containing $3 \%(\mathrm{w} / \mathrm{v})$ sugar and $0.8 \%(\mathrm{w} / \mathrm{v})$ agar and growth conditions were as follows: $16 \mathrm{~h}$ light $/ 8 \mathrm{~h}$ dark cycle under $25^{\circ} \mathrm{C}$ with $60 \%$ relative air humidity as reported before $[3,4,21]$. Seeds of $N$. benthamiana were sown and cultivated in the pots supplemented with soil matrix for 4-5 weeks for infiltration [15,32] and all plants were maintained in the greenhouse.

All strains and plasmid vectors used in this study are part of the collection that is available in our laboratory. The intermediate cloning vector pMD-18 T and reverse transcriptase M-MLV were purchased from TaKaRa Biotechnology (Dalian, China) Limited Company. Primer synthesis and DNA sequencing were performed by Shanghai Sangon Biotechnological Company, China. The RNA extraction kit and qRTPCR kit were purchased from Tiangen Biotechnology (Beijing) Limited Company, China. Standards of cryptotanshinone, tanshinone I, tanshinone IIA, dihydrotanshinone used for HPLC analysis were purchased from Aladdin, China.

\subsection{Elicitor preparation}

For MeJA induction, the MeJA (bought from Sigma) was firstly dissolved in a small volume of ethanol, and then dissolved into distilled water to a storage concentration of $50 \mathrm{mM}$. A final working concentration of $100 \mu \mathrm{M}$ MeJA was employed for the elicitation assay, and an equivalent volume of sterilized water was used as the mock treatment $[9,37]$. Time points of analysis were selected at $0,0.5,1,2,4 \mathrm{~h}$ after MJ treatment to monitor the effects of MeJA to induce metabolite biosynthesis.

\subsection{Identification and cloning of SmWRKY2}

A local reference transcript database of $S$. miltiorrhiza built up as reported previously was used for this research [11]. We obtain the fulllength cDNA sequence of the putative SmWRKY2 gene. Primer pairs for $S m W R K Y 2-K F$ and SmWRKY2-KR were synthesized for amplification of the full-length ORF of SmWRKY2 according to the procedure as described below: initial denaturation at $94^{\circ} \mathrm{C}$ for $10 \mathrm{~min}, 35$ cycles of $94^{\circ} \mathrm{C}$ for $30 \mathrm{~s}, 55^{\circ} \mathrm{C}$ for $30 \mathrm{~s}$ and $72^{\circ} \mathrm{C}$ for $90 \mathrm{~s}$, followed by a final extension at $72{ }^{\circ} \mathrm{C}$ for $10 \mathrm{~min}$. All primers used for identification of SmWRKY2 are listed in Supplemental Table 1.

\subsection{Bioinformatics analysis of SmWRKY2}

Characterization of SmWRKY2 were further analyzed by a series of bioinformatics tools. Nucleotide blast, protein blast and ORF Finder were used to assess nucleotide sequences and complete open reading frame (ORF) of the gene of interest. MEGA 6 was applied to construct a phylogenetic tree by the neighbor-joining (NJ) method and 1000 replications were performed for bootstrap values. Multiple sequence alignment of SmWRKY2 with other WRKYs were carried out using Clustal X with default parameters [32,38]. The isoelectric point was predicted online (https://web.expasy.org/protparam/).

\subsection{Expression pattern of SmWRKY2}

Different tissues including the tap root, stem, leaf, flower and seed were gathered from two-year-old $S$. miltiorrhiza plants. Elicitor treatments were conducted on $S$. miltiorrhiza hairy roots derived from Ri plasmid harbouring C58C1 strain of Agrobacterium tumefaciens transformation that are routinely subcultured every 60 days. Hairy roots were harvested at selected time points $(0,0.5,1,2$ and $4 \mathrm{~h})$ after MJ 
treatment. All the treated samples were immediately frozen in liquid nitrogen and stored at $-80^{\circ} \mathrm{C}$ before the analysis of the expression profiles of SmWRKY2 in generated transgenic lines.

\subsection{Subcellular localization of SmWRKY2}

To analyze the subcellular localization of SmWRKY2, PCR products of the full-length of SmWRKY2 with Hind III and Spe I restriction sites were cloned into the vector pHB-YFP to generate the recombinant vector pHB-SmWRKY2-YFP. The constructed expression vector was transferred into Agrobacterium tumefaciens strain GV3101 and injected into forty-day-old tobacco leaves [29,32,38]. DAPI solution of $1 \mu \mathrm{gL}^{-1}$ was injected into $N$. benthamiana leaves $3 \mathrm{~h}$ before observation. YFP fluorescence was observed after $48 \mathrm{~h}$ cultivation using a confocal microscope (Carl Zeiss).

\subsection{Generation of transgenic SmWRKY2 hairy roots}

The full-length coding sequence of SmWRKY2 with restriction sites, SpeI and BstE II, was cloned and inserted into a modified pCAMBIA2300 $^{\mathrm{sm}}$ vector (where the small fragment digested by EcoR I and Hind III is replaced with the corresponding GFP-GUS gene expression cassette from pCAMBIA1304) under the control of the CaMV 35S promoter to generate pCAMBIA2300 ${ }^{\mathrm{sm}}$-SmWRKY2 as described before (Fig. S2) [11,38]. The disarmed A. tumefaciens strain C58C1 harboring the $A$. rhizogenes Ri plasmid pRiA4 and pCAMBIA2300 $0^{\mathrm{sm}}-S m W R K Y 2$ was used to infect the aseptic explants, generated via tissue culture, and the empty pCAMBIA2300 $0^{\mathrm{sm}}$ was regarded as the control. The transformation procedure was the same as our previous study [3,4,11,38]. Those hairy roots that showed a good hairy root phenotype were subcultured and primer pairs CaMV35S-F23 and SmWRKY2-QR were used to identify the positive lines by polymerase chain reaction (PCR) analysis, and the rol B gene was also detected. Genomic DNA was extracted from hairy root lines using the cetyltrimethyl ammonium bromide (CTAB) method as reported previously [32,39]. Identified positive clones were cut into segments that were approximately $4 \mathrm{~cm}$ long for shake-flask culture in $100 \mathrm{~mL} 1 / 2 \mathrm{MS}$ medium and these were cultured at $25^{\circ} \mathrm{C}$ on an orbital shaker shaking at the speed of $100 \mathrm{rpm}$ in the dark $[15,38]$. Primers sequences used for amplifications are listed in Supplemental Table 1.

\subsection{Total RNA isolation and relative expression analysis via $q R T-P C R$}

Expression profiles of SmWRKY2 and several key enzyme genes involved in the tanshinone biosynthetic pathway were investigated by real-time quantitative PCR analysis (qRT-PCR). Total RNA was extracted from different tissues with the RNA prep pure plant kit as described before $[3,15]$. Total RNA served as the template for reverse transcription (RT) reaction, the reaction conditions were according to our previous studies [9,15,38]. Gene-specific primers (listed in Supplemental Table 1) for qRT-PCR were designed to assay the relative expression level and were compared with an internal reference gene SmActin, using the relative quantitative analysis method $\left(2-^{\Delta \Delta \mathrm{CT}}\right)$. Amplifications were performed according to the manufacturer's instructions: one cycle of denaturation at $95^{\circ} \mathrm{C}$ for $15 \mathrm{~min}$, then 40 cycles of $15 \mathrm{~s}$ denaturation at $95^{\circ} \mathrm{C}, 30 \mathrm{~s}$ annealing at $60^{\circ} \mathrm{C}$ and $30 \mathrm{~s}$ extension at $72{ }^{\circ} \mathrm{C}[11,21]$.

\subsection{Dual-Luciferase (Dual-LUC) assay}

For the dual-LUC assay, the promoters of SmDXS2 and SmCPS with $K p n I$ and $X h o I$ restriction sites were cloned into pGREEN0800 to drive the luciferase reporters, respectively. And the complete ORF of $S m W R K Y 2$ was inserted into the $p H B-Y F P$ vector as effector. The $p H B$ SmWRKY2-YFP and $p H B$-YFP empty plasmid were transferred into Agrobacterium tumefaciens strain GV3101 individually. The
pGREEN0800-pSTDXS2, pGREENO800-pSTCPS was separately cotransformed with the helper plasmid pSoup19 into GV3101, and the assay was conducted as described before [11,21,29]. The $p H B-Y F P$ empty plasmid was used as a negative control. The $35 \mathrm{~S}$ promoter-driven Renilla was taken as an internal control. Each sample were measured for three biological times. The reporter strain with effector strain was mixed with ratio of one-to-one to inject the tobacco leaves. After two days' injection, the samples were collected for dual-LUC assay by reaction reagents according to the manufacturer (Promega).

\subsection{Yeast one-hybrid assay}

We performed yeast one-hybrid assay as reported before [29]. The coding sequence of full-length SmWRKY2 cDNA was fused into the $p B 42 \mathrm{AD}$ vector. SmCPS promoter sequence (from $-323 \mathrm{bp}$ to $-329 \mathrm{bp}$, relative to translation start site) was inserted into a $p L a c Z$ plasmid. The resulting recombinant plasmids were co-transformed into yeast strain EGY48a. The transformants were cultivated on SD/-Ura/-Trp medium for two days, and tested on SD/-Ura/-Trp medium with 5-bromo-4chloro-3-indolyl-b-D-galactopyranoside (X-gal) for $24 \mathrm{~h}$. The empty vectors $p B 42 \mathrm{AD}$ and $p L a c Z$ were used as negative controls.

\subsection{Electrophoretic mobility shift assay (EMSA)}

The ORF of SmWRKY2 was inserted into the expression vector pET28a between the NcoI and SalI sites. Recombinant SmWRKY2 was expressed in Escherichia coli strain BL21, and purified using a $6 \times$ HISTagged Protein Kit (Cwbio, Shanghai, China). Oligonucleotide probes for the SmCPS promoter was labeled with an EMSA probe biotin labeling kit (Beyotime, Haimen, China), following the manufacturer's instructions. The DNA fragment and purified proteins were incubated in $10 \times$ EMSA binding buffer ( $2 \mathrm{mM}$ DTT, $0.2 \mathrm{mM}$ EDTA, $50 \mathrm{mM}$ HEPES$\mathrm{KOH}$ at $\mathrm{pH} 7.6,300 \mathrm{mM} \mathrm{NaCl}, 100 \mathrm{ng} \mu \mathrm{L}^{-1}$ poly (dI-dC), and $400 \mathrm{ng}$ $\mu \mathrm{L}^{-1}$ salmon sperm DNA) at $25^{\circ} \mathrm{C}$ for $20-25$ min. The DNA-protein complexes were then electro-transferred and detected with a Chemiluminescent Nucleic Acid Detection Module (Thermo Fisher Scientific, Shanghai, China), following the manufacturer's instructions. Fluorescence was detected with a c300 image scanner (Azure Biosystems, USA). The probe sequences are listed in Supplemental Table 1.

\subsection{HPLC analysis of tanshinones}

The 60-day-old hairy roots were dried at $50{ }^{\circ} \mathrm{C}$ to a constant weight in an oven. For each analysis, approximately $100 \mathrm{mg}$ dried hairy roots were ground into a powder and immersed in $16 \mathrm{~mL}$ methanol/dichloromethane $(3: 1, \mathrm{v} / \mathrm{v})$ for tanshinone extraction. Tanshinone extractions were carried out as reported before [4,9,11]. HPLC analysis was performed on an Agilent 1260 apparatus equipped with a Waters reversed-phase C18 symmetry column, and the detection conditions were performed following the methods described previously [3,15,38], which worked as the mobile phase at a flow rate of $1 \mathrm{~mL} / \mathrm{min}$ with the detection wavelength at $270 \mathrm{~nm}$. Total tashinones was regarded as TT in this study.

\subsection{Statistical analysis}

All the experiments including culture of hairy root clones, PCR identification, qRT-PCR and HPLC analysis were repeated three times. Results of tanshinone content were presented as mean values \pm SD. The error bars were from biological triplicates. The statistically significant difference was analyzed by one sample $t$ test, and the difference of various hairy root lines were used in the one-way analysis of variance (ANOVA) using SPSS 11.5 software (SPSS, Inc.). 
(a)

\begin{tabular}{|c|c|}
\hline AtWRKY32 & -VSVPTKQEQRSDSPVVNRLSVTPVPRTPARDGYNWRKYGQKQVKSPKGSRSY \\
\hline AtWRKY1 & PKVSE-_-_-SSGALSLQSGSEGNSPFIREKVMEDGYNWRKYGQKLVKGNEFVRSY \\
\hline AtWRKY58 & -HYNNPGNNNNNRSYNVVNVDKPADDGYNWRKYGQKPIKGCEYPRSY \\
\hline SmWRKY2 & -ESSDFSQSDQKFQPANFAVDKPTDDGYNWRKYGQKQVKGSEFPRSY \\
\hline AtWRKY4 & -TSDVTIIEHR-SQQPLNVDKPADDGYNWRKYGQKQVKGSEFPRSY \\
\hline AtWRKY3 & --TSEISVFEHR-S-QPQNADKPADDGYNWRKYGQKQVKGSDFPRSY \\
\hline KKY44 & - -VQKVPSFTESETSTGDRSSVDGYNWRKYGQKQVKGSECPRSY \\
\hline YY20 & P--TSSDIPAGSDQEESIQTSQNDSRGSTPSILADDGYNWRKYGQKHVKGSEFPRSY \\
\hline iY25 & IPNEIAT-----QNNNQSFGT-ERQIKIPAYMVSRNSNDGYGWRKYGQKQVKKSENPRSY \\
\hline AtWRKY2 & KTSDVTTNTSLETVDH-QEEEEEQRRGDSMAGGAPAEDGYNWRKYGQKLVKGSEYPRSY \\
\hline Y34 & -TNIALEPDDSQDYEERQLGGLGDSMACCAPADDGYNWRKY \\
\hline Y33 & --WSQTETRPNNQAVSYNGREQRKGEDG \\
\hline \multirow[t]{2}{*}{ AtWRKY26 } & -SEIMSSNKTSDDGYNWRKYGQKQVKGSENPRSY \\
\hline & $* * *, * *$ \\
\hline & NSQ-SSDSVSKPGKKNKFVVHAAGDVGICGDGYRWRKYGQKMVK \\
\hline & PRSYYRCSS \\
\hline $\mathrm{Y} 58$ & IPRSYYKCTT \\
\hline SmWRKY2 & YPRSYYKCTT \\
\hline & PYPRSYYKCTT \\
\hline RKY3 & EVRVSEPV-ASSHRTVTEPRIIVQTTSEVDLLDDGYRWRKYGQKVVKGNPYPRSYYKCTT \\
\hline KY44 & EKQ—— SSEAGVSQGSVESDSLEDGFRWRKYGQKVVGGNAYPRSYYRCTS \\
\hline & NPRSYYKCTA \\
\hline & NPRSYYKCTF \\
\hline & NPRSYYKCTA \\
\hline AtWRKY34 & -YATE-TSGSTRASREPRVVVQTTSDIDILDDGYRWRKYGQKVVKGNPNPRSYYKCTA \\
\hline AtWRKY33 & DNETNGG-NGGGSKTVREPRIVVQTTSDIDILDDGYRWRKYGQKVVKGNPNPRSYYKCTT \\
\hline AtWRKY26 & ENVKEPRVVVQTTSDIDILDDGYRWRKYGQKVVKGNPNPRSYYKCTF \\
\hline
\end{tabular}

Fig. 1. Bioinformatics analysis of SmWRKY2.

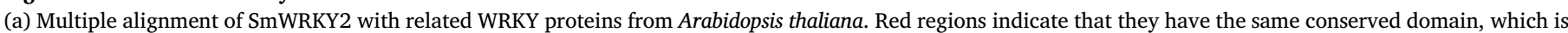

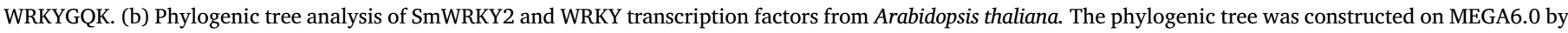

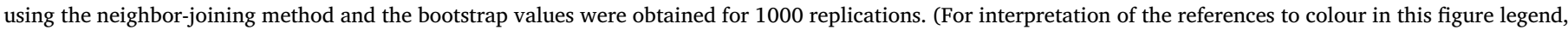
the reader is referred to the web version of this article)

\section{Results}

\subsection{Isolation and characterization of SmWRKY2}

By searching our local transcriptome database [19], one WKRY showing highly homology to WRKY4 from Arabidopsis thalianas (AtWRKY4) was cloned for further research. The putative SmWRKY2 ORF contains $1476 \mathrm{bp}$ and encodes a protein of 491 amino acids with a calculated molecular mass of 53.3KD and $\mathrm{pI}$ of $\mathrm{pH}$ 7.26. To further figure out the biological characteristics and phylogenetic relationship of SmWRKY2, a series of bioinformatic analyses were performed. Multiple alignment of SmWRKY2 with related WRKY proteins from A. thaliana revealed that SmWRKY2 contained two conserved WRKY domain (WRKYGQK) and a special zinc finger like motif in its C-terminal which falls into the group I of WRKY family (Fig. 1a). Phylogenetic analysis illustrated that SmWRKY2 has a close relationship with AtWRKY4 (Fig. 1b).

\subsection{Expression patterns of SmWRKY2}

To investigate the tissue expression pattern of SmWRKY2, different tissues including root, stem, leaf, flower and seed from two-year-old $S$. miltiorrhiza plants were analyzed. SmWRKY2 showed high expression in leaf and stem tissues, and relatively low expression level in the flower and roots, plus its transcript was barely detected in seed (Fig. 2a). This result indicated that SmWRKY2 does not exhibit a tissue-constitutive gene expression. The elicitation results indicated that SmWRKY2 expression was induced by exogenous MeJA with the expression levels reaching a peak at $1 \mathrm{~h}$ after treatment, leading to an approximate 2-fold increase compared with control. This was followed by another increase at $4 \mathrm{~h}$ (Fig. 2b), suggesting that SmWRKY2 may participate in MJ signalling.

\subsection{Subcellular localization of SmWRKY2}

To experimentally confirm the subcellular localization of

\section{(b)}

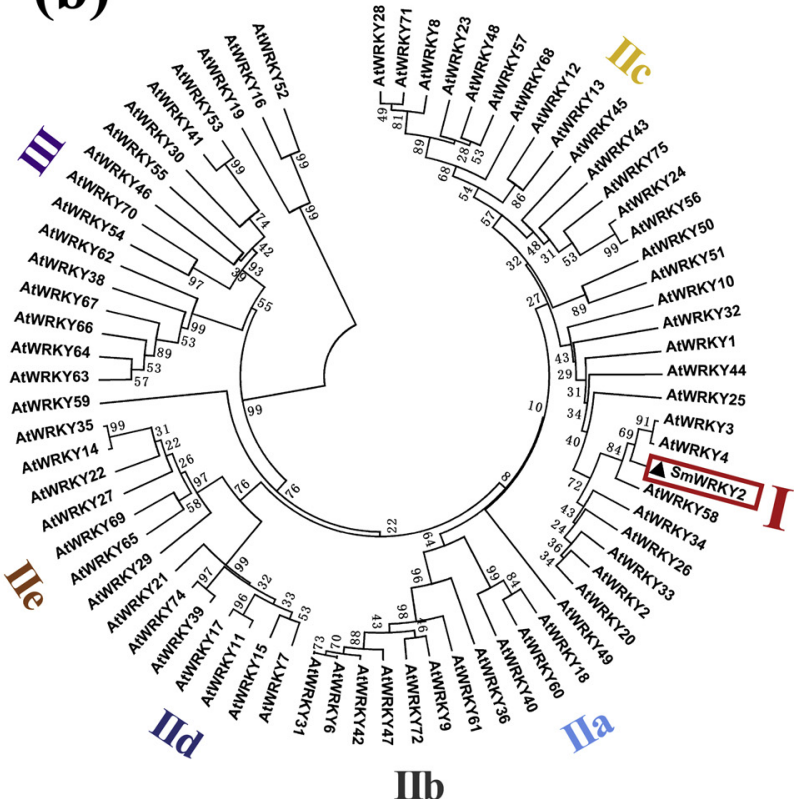

SmWRKY2, SmWRKY2 was cloned into the pHB vector fused with a yellow fluorescent protein (YFP) reporter gene to generate the vector pHB-SmWRKY2-YFP. Then, the constructed vector and the pHB-YFP (used as the control) was transformed into GV3101 strain and expressed in tobacco leaves, respectively. Fluorescence of YFP was detected in the cytoplasm and nucleus in the tobacco leaves transformed with the empty vector. Contrast to this, the fluorescent signal of SmWRKY2 was only detected in the nucleus (Fig. 3). The expression pattern was consistent with the characteristic mode of action of SmWRKY2 as a transcription factor.

\subsection{Generation of SmWRKY2 transgenic hairy roots}

To further investigate the function of SmWRKY2 in S. miltiorrhiza, we inserted SmWRKY2 into a modified pCAMBIA2300 ${ }^{\mathrm{sm}}$ vector. Afterwards, the recombinant overexpression vector pCAMBIA2300 ${ }^{\mathrm{sm}}$ SmWRKY2 was introduced into the modified Agrobacterium tumefaciens stain $\mathrm{C} 58 \mathrm{C} 1$ and used to infect $S$. miltiorrhiza explants, while the empty vector pCAMBIA2300 $0^{\mathrm{sm}}$ was used as control. After the infection process, fresh hairy roots could be induced from stem or leaf explants (Fig. 4a). The positive lines carrying the SmWRKY2 gene (OE line) were verified by genomic PCR and a positive transformation rate of $26.92 \%$ among the 21 hairy root lines was obtained. And $50 \%$ positive rate of antisense lines were generated. Three individual over-expressed (OE-1, 2, 3 ) and two anti-sense lines (Anti-sense-1, 2) were chosen for further study (Fig. 4b). qRT-PCR analysis of the expression of SmWRKY2 in overexpression lines revealed that SmWRKY2 was expressed 5 to 65fold higher than in the control lines, while the expression is slightly lower in the Anti-SmWRKY2 lines than the controls (Fig. 5a).

\subsection{SmDXS2 and SmCPS were significantly up-regulated by SmWRKY2 in} vivo

To study whether SmWRKY2 participated in the regulation of tanshinone biosynthesis, transcript levels of several genes related to tanshinones biosynthesis in SmWRKY2 transgenic hairy root were analyzed 
(a)

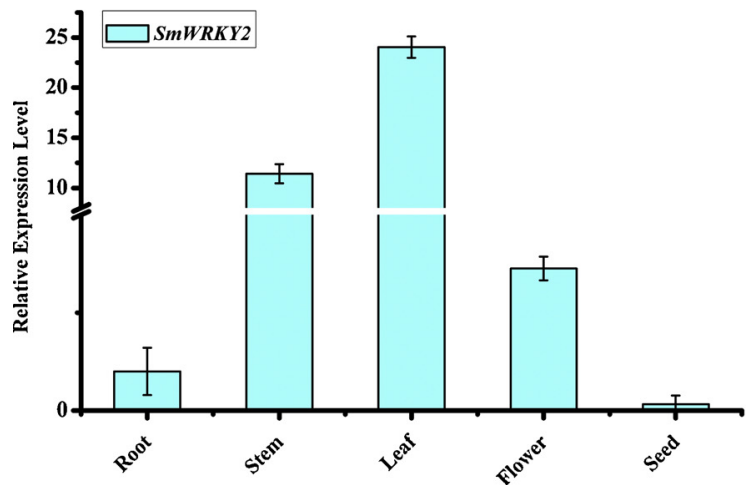

(b)

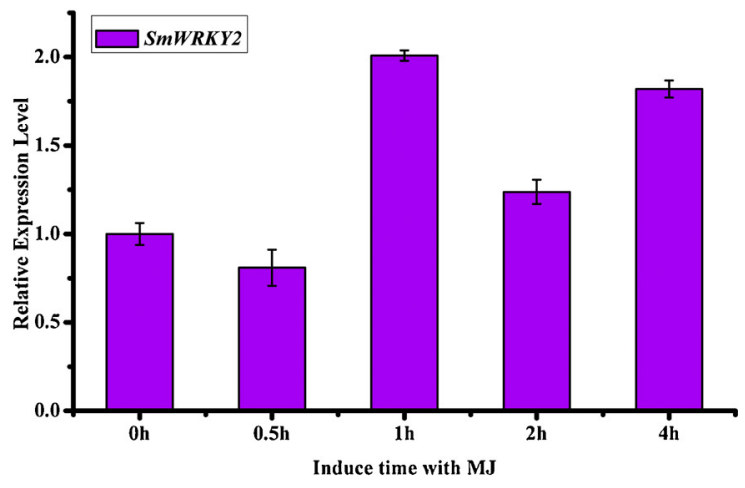

Fig. 2. Expression pattern of SmWRKY2.

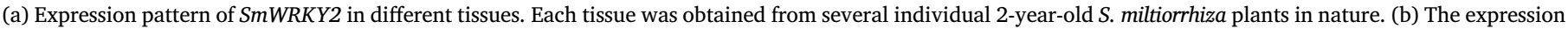

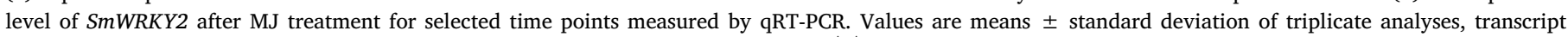
abundance of SmWRKY2 is normalized to the internal standard SmActin using the $2^{-\triangle \triangle \mathrm{CT}}$ method.

by qRT-PCR. Several tanshinone biosynthesis pathway genes were upregulated in the SmWRKY2-overexpressing hairy roots, the most striking ones were SmDXS2 and SmCPS, resulting in 10-15 folds and 5-25 folds increases compared with the control, respectively. Meanwhile the expression of SmIPPI, SmGGPPS, SmKSL and SmCYP76AH1 was also increased by about 2-4 folds in overexpression lines than the control, whereas, only CPS was slightly downregulated in antisense lines (Fig. 5b). Dual-Luciferase (Dual-LUC) assay showed that SmWRKY2 can elevated the expression of SmDXS2 and SmCPS about 2-3 folds. The data revealed that SmWRKY2 may be a regulator in tanshinone biosynthesis by positively activating the expression of SmDXS2 and SmCPS (Fig. 6b, Fig. S3b).

\subsection{Activation of the expression of SmCPS by SmWRKY2}

To investigate further how SmWRKY2 regulates tanshinone biosynthesis, we performed Dual-LUC assays. The result showed that SmWRKY2 activated the transcription of SmDXS2 and SmCPS, But the $S m D X S 2$ slightly up-regulated. Meanwhile, analyzed the SmDXS2 and SmCPS promoter sequences and found that they all have W-box (Figs. 6a, S3a). Y1H assay showed that SmWRKY2 was bound to the Wbox of SmCPS promoter (Fig. 6c). We performed an EMSA using purified His-SmWRKY2 recombinant protein and biotin-modified oligonucleotide probes containing the W-box sequences to examine the binding of SmWRKY2 to the SmCPS promoter (Fig. 6d). Our result indicated that SmWRKY2 could bind to the W-box element of the SmCPS promoter region, which was consistent with that SmCPS expression was effectively activated in SmWRKY2-overexpressing S. miltiorrhiza hairy roots.

\subsection{Accumulation of tanshinone was obviously affected by SmWRKY2}

Four kinds of tanshinones including cryptotanshinone, dihydrotanshinone I, tanshinone I, tanshinone IIA were examined in hairy roots by HPLC. The results showed that the content of total tanshinones were significantly up-regulated from 3.003 to $3.985 \mathrm{mg} / \mathrm{g}$ DW in over-expressed lines. Among them, OE-SmWRKY2-1 line accumulated the highest content of total tanshinone, which was 1.83 times as that of the control. The total tanshinone content in the two antisense-SmWRKY2 lines was slightly lower than the control group (Fig. 7). These results further confirmed the positive role of SmWRKY2 in the regulation of tanshinone biosynthesis.

\section{Discussion}

S. miltiorrhiza is a traditional Chinese herbal medicine that has been used for more than two thousand years [14]. Previous studies have shown that tanshinones are the main medicinal active ingredient of $S$. miltiorrhiza that can be used to treat cardiovascular and cerebrovascular diseases [31]. However, traditional S. miltiorrhiza production cannot meet the growing clinical needs due to its slow growth, low tanshinone content and scarcity of wild resources [32] and for these reasons biotechnological approaches may provide alternative means for tanshinone production. The diterpenoid tanshinone was synthesized in

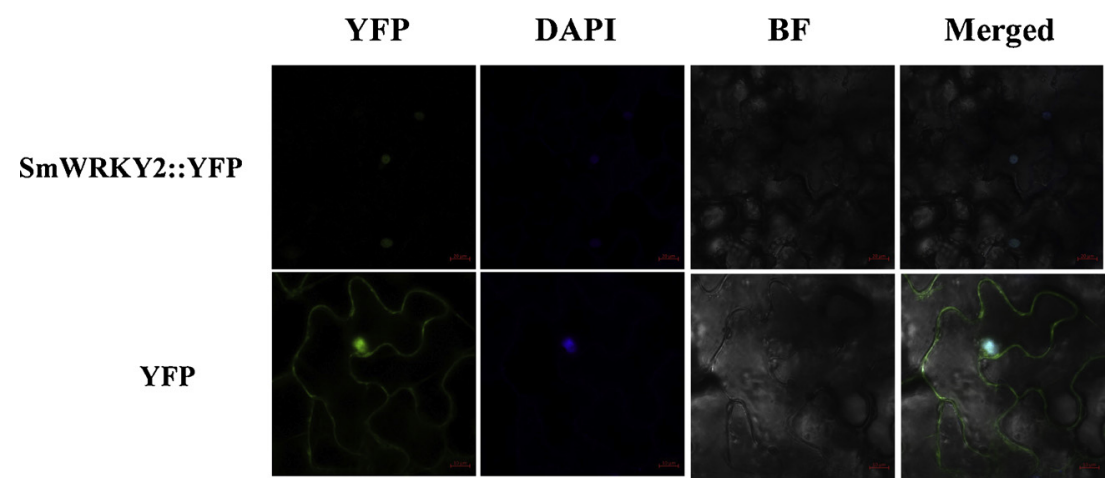

Fig. 3. Subcellular localization of SmWRKY2.

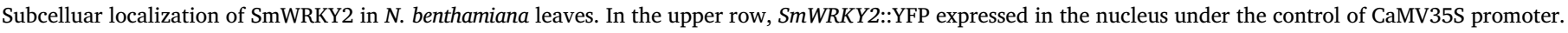
In the lower row, YFP control expressed in the whole $N$. benthamiana leaf cell. 
(a)

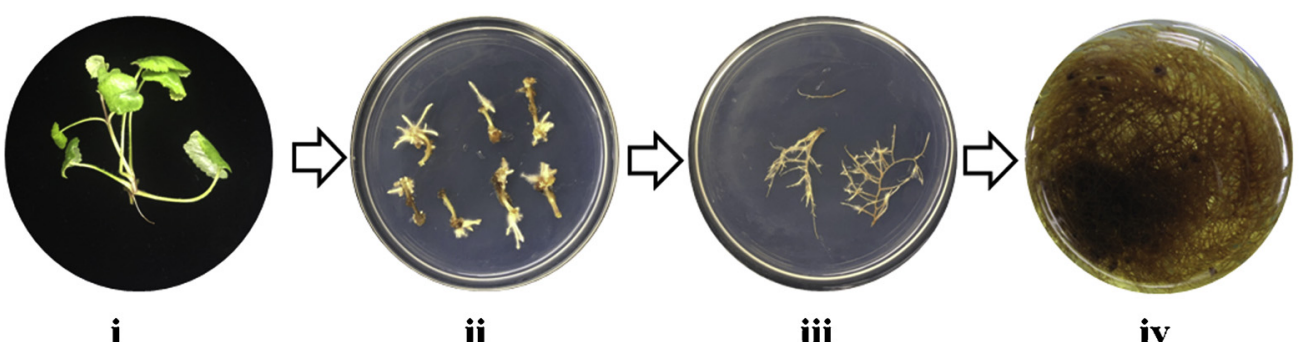

(b)

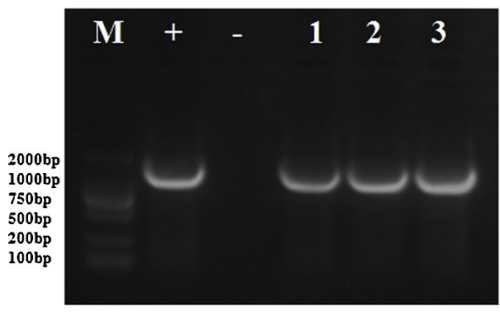

OE-SmWRKY2

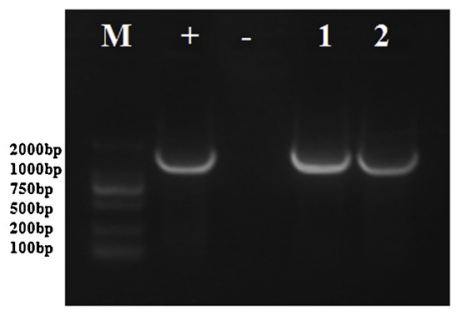

Anti-SmWRKY2

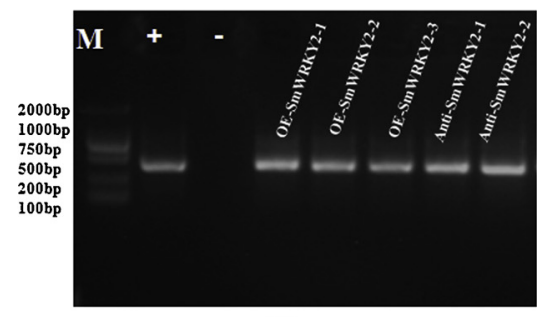

rolB

Fig. 4. Acquisition and identification of hairy roots transgenic hairy roots lines.

(a) Generation of transgenic hairy root of $S$. miltiorrhiza. i) S. miltiorrhiza seedlings in MS medium; ii) growing hairy roots on the infected $S$. miltiorrhiza explants; iii) a single clone of a hairy root line; and iv), hairy root cultured in 1/2 MS medium. (b) Identification of positive transgenic hairy root lines by PCR.

distinct pathways including the MVA and MEP pathways, and several key genes have been isolated in Danshen. For example, DXS catalyzes the first step in MEP pathway by metabolizing pyruvate and glyceraldehyde 3-phosphate to form DXP, which has been validated as a ratelimiting process [3]. RNA interference of SmCPS expression resulted in reduced tanshinone content in transgenic hairy roots [33], suggesting that SmCPS is a key enzyme in tanshinone biosynthesis. Genetic engineering of key enzyme genes individually or in combinations has become an effective and important way to increase the accumulation of active ingredients in S. miltiorrhiza [3]. For example, introduction of single SmDXS2 gene in danshen hairy roots produced tanshinone quantities ranging from $2.02-4.58 \mathrm{mg} / \mathrm{g}$ DW, which was much higher than the control line [11]. Co-expression of SmGGPPS and SmDXS2 led to superior tanshinone production, recorded at $12.93 \mathrm{mg} / \mathrm{g}$ DW [11].

WRKY is one of the largest plant transcription factor families that have been studied recently $[28,30]$. The conserved domain WRKYGQK and the zinc finger motif consisting of 60 amino acids is considered as a

(a)

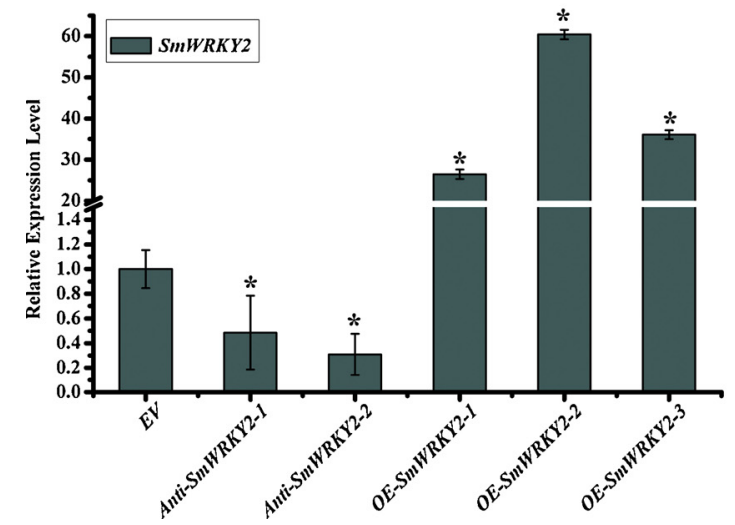

general feature of WRKY transcription factors and can also serve as a standard means to identify this subgroup. Previous studies have demonstrated that WRKY transcription factors can directly bind to W-box of related genes from different signaling pathways and play a regulatory role in plant stress tolerance [23,26,27]. For example, SpWRKY1 has been shown to promote resistance to Phytophthora nicotianae and tolerance to salt and drought stress in transgenic tobacco [25]. Overexpression of NtWRKY5O increased the expression level of related defense genes and improve resistance to Ralstonia solanacearum in tabacoo [34]. GhWRKY25 (member of Group I) from cotton confers differential tolerance to non-biological and biotic stress in transgenic tobacco [27]. In recent years, there is increasing evidence that WRKY transcription factors are not only involved in plant stress resistance, but also in plant secondary metabolism regulation $[28,30]$. For example, Taxus chinensis WRKY1 may enhance the expression of 10-deacetylbaccatin III-10 $\beta$-Oacetyl transferase (DBAT) to increase the accumulation of taxol in Taxus cell cultures [35]. A WRKY gene named WRKY1 from Withania

\section{(b)}

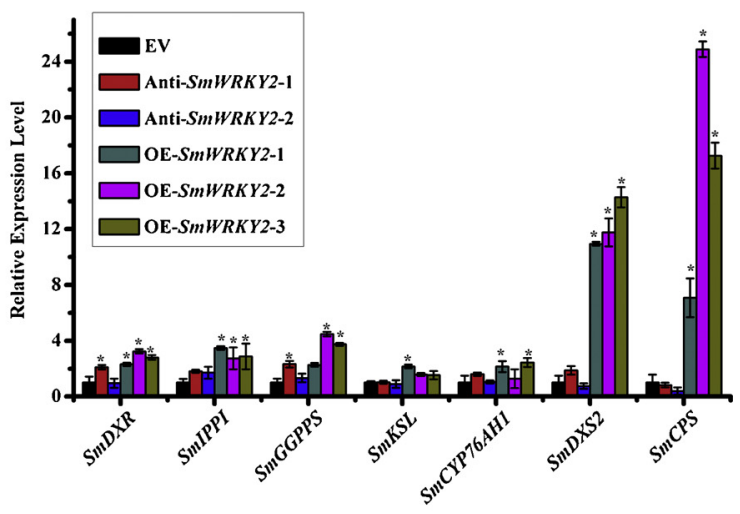

Fig. 5. Detection of transcript level of rate-limiting enzyme genes.

(a) Transcript levels of SmWRKY2 in transgenic hairy roots detected by qRT-PCR. (b) Transcript levels of key tanshinone biosynthesis genes in SmWRKY2 transgenic hairy roots, indicating that SmDXS2 and SmCPS are significantly up-regulated. All data are means of three replicates, with error bars indicating standard deviations. The values are means \pm S.D of triplicate analysis $(\mathrm{P}<0.05)$. 
(a)

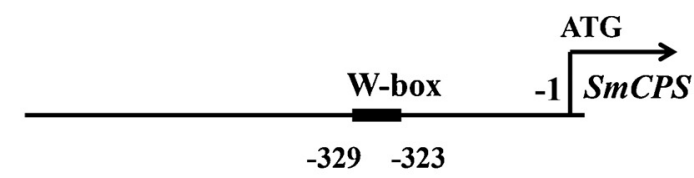

(c)

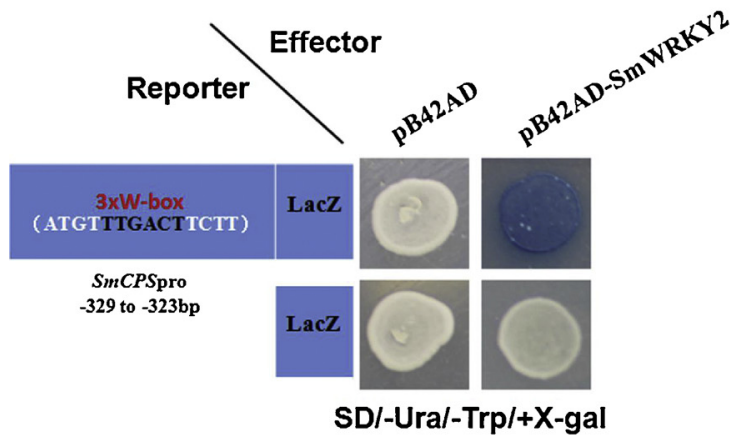

(b)
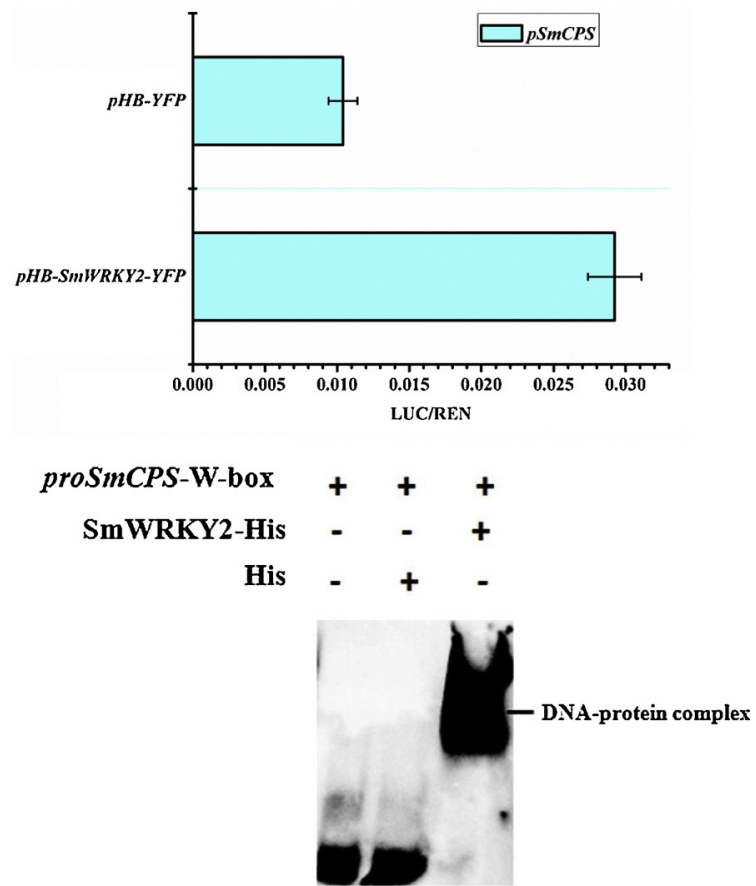

Fig. 6. SmWRKY2 activates the transcription of SmCPSin vivo.

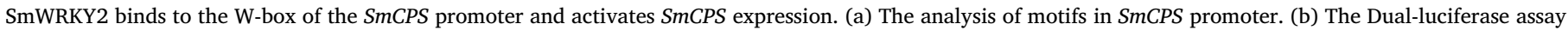

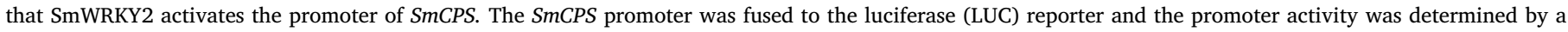

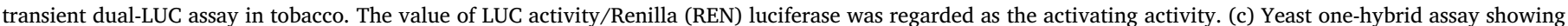

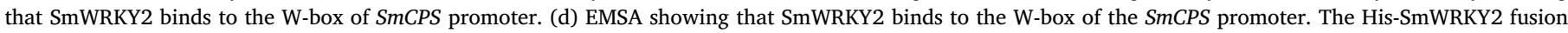
protein and labeled probe were used. The HIS protein was included as a negative control. Error bars indicate SD ( $=3$ ).

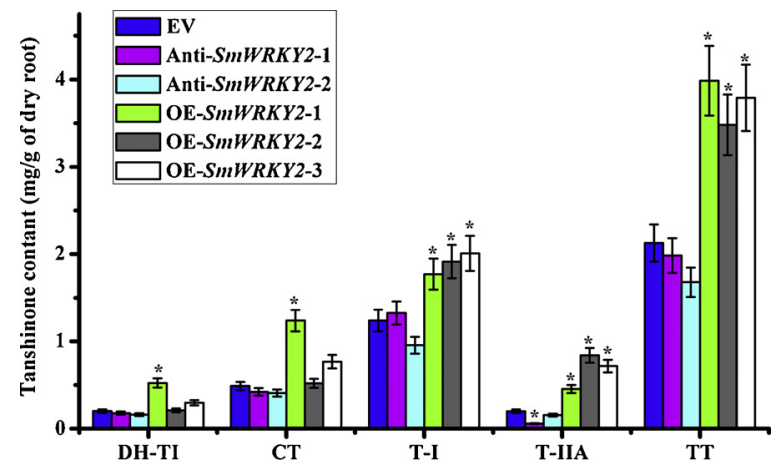

Fig. 7. HPLC analysis of tanshinones in transgenic lines.

The production of tanshinone in SmWRKY2 transgenic hairy roots compared with control line detected by HPLC. Three overexpressed lines (OE-SmWRKY2$1,2,3$ ) and two antisence lines (Anti-SmWRKY2-1, 2) were used to compare with the control hairy root line. DH-TI, dihydrotanshinone; CT, cryptotanshinone; T-I, tanshinone I; T-IIA, tanshinone IIA; TT represented total tanshinone. All data are means of three replicates, with error bars indicating standard deviations. The values are means \pm S.D of triplicate analysis $(\mathrm{P}<0.05)$

somnifera could target squalene synthase and squalene epoxidase gene for improvement of triterpenoid withanolide accumulation [36]. However, studies on the function of WRKY transcription factors in $S$. miltiorrhiza regulating the biosynthesis of tanshinones are relatively rare.

In our study, a novel WRKY transcription factor SmWRKY2 was successfully cloned from $S$. miltiorrhiza, with high homology to AtWRKY4 factor from Arabidopsis. In S. miltiorrhiza, exogenous MeJA treatment can promote the accumulation of tanshinone [9,37]. In our study, we noted that SmWRKY2 can be induced by exogenous MeJA treatment, reaching high levels of accumulation $1 \mathrm{~h}$ after the MeJA treatment, which is consistent with previous reports that MeJA treatment can increase tanshinone production $[9,19]$. Analysis using HPLC showed that the introduction of SmWRKY2 in transgenic hairy roots increased the tanshinone to $3.985 \mathrm{mg} / \mathrm{g} \mathrm{DW}$, which was 1.83 times as that of the non-transgenic line. Quantification via qRT-PCR analysis showed that SmWRKY2 overexpression significantly promoted the transcript levels of SmDXS2 and SmCPS which are two key genes in tanshinone biosynthesis, dual-Luciferase (dual-LUC) assay showed that SmWRKY2 can positively regulate SmDXS2 and SmCPS expression, Y1H and EMSA experiments indicate that SmWRKY2 only binds to the Wbox of the SmCPS promoter, suggesting the regulatory role of SmWRKY2 in tanshinone synthesis in $S$. miltiorrhiza. The expression profile showed that SmWRKY2 responded to MeJA, suggesting that SmWRKY2 may be involved in stress-regulating processes, such as defense against pathogens, but further experimental confirmation is needed to verify this idea. In summary, our work reveals that transcription factor SmWRKY2 is involved in promotion of tanshinone biosynthesis and provides new insights into the potential of using a genetic engineering strategy to increase the yield of target compounds in $S$. miltiorrhiza.

\section{Author contributions}

G.Y.K. conceived and designed the experiments. C.P.D., X.L.H., M.S., R.F., Y.W., Y.Z., and Y.F. performed the experiments. C.P.D, X.L.H. and W.Z. analyzed the data. M.S., X.L.H., C.P.D., N.P.M. and G.Y.K. wrote the paper.

\section{Conflicts of interest}

The authors declare no conflict of interest. 


\section{Acknowledgements}

This work was supported by the National Natural Science Fund (81522049, 31571735, 31270007), Shanghai Science and Technology Committee Project (17JC1404300, 15430502700), Zhejiang Provincial Ten Thousands Program for Leading Talents of Science and Technology Innovation, Zhejiang Provincial Program for the Cultivation of Highlevel Innovative Health talents and Opening Fund of Zhejiang Provincial First-Class Discipline for TCM (ZYAOX2018005, ZYAOX2018035), New Century Talent Project (NECT-13-0902) and the "Dawn" Program of Shanghai Education Commission (16SG38). NPM is funded by the National Research Foundation of South Africa (Pretoria) (109385) and Stellenbosch University Division of Research Development is thanked for incentive funding this collaboration.

\section{Appendix A. Supplementary data}

Supplementary material related to this article can be found, in the online version, at doi:https://doi.org/10.1016/j.plantsci.2019.03.007.

\section{References}

[1] K. Landmark, Acetylsalicylic acid in the treatment of cardiovascular and cerebrovascular diseases, Tidsskr. Nor. Laegeforen. 117 (1997) 4432-4436.

[2] Y. Zhang, X. Li, Z. Wang, Antioxidant activities of leaf extract of Salvia miltiorrhiza Bunge and related phenolic constituents, Food Chem. Toxicol. 48 (2010) 2656-2662.

[3] G.Y. Kai, H. Xu, C.C. Zhou, P. Liao, J.B. Xiao, X.Q. Luo, L.J. You, L. Zhang, Metabolic engineering tanshinone biosynthetic pathway in Salvia miltiorrhiza hairy root cultures, Metab. Eng. 13 (2011) 319-327.

[4] M. Shi, X.Q. Luo, G.H. Ju, X.H. Yu, X.L. Hao, Q. Huang, J.B. Xiao, L.J. Cui, G.Y. Kai, Increased accumulation of the cardio-cerebrovascular disease treatment drug tanshinone in Salvia miltiorrhiza hairy roots by the enzymes 3-hydroxy-3-methylglutaryl CoA reductase and 1-deoxy-D-xylulose 5-phosphate reductoisomerase, Funct. Integr. Genomics 14 (2014) 603-615.

[5] N. Yang, W.P. Zhou, J. Su, X.F. Wang, L. Li, L.R. Wang, X.Y. Cao, Z.Z. Wang, Overexpression of SmMYC2 increases the production of phenolic acids in Salvia miltiorrhiza, Front. Plant Sci. 8 (2017) 1804.

[6] P. Liao, W. Zhou, L. Zhang, J. Wang, X.M. Yan, Y. Zhang, R. Zhang, L. Li, G.Y. Zhou, G.Y. Kai, Molecular cloning, characterization and expression analysis of a new gene encoding 3-hydroxy-3-methylglutaryl coenzyme A reductase from Salvia miltiorrhiza, Acta Physiol. Plant. 31 (2009) 565-572.

[7] M. Shi, F.F. Huang, C.P. Deng, Y. Wang, G.Y. Kai, Bioactivities, biosynthesis and biotechnological production of phenolic acids in Salvia miltiorrhiza, Crit. Rev. Food Sci. Nutr. (2018), https://doi.org/10.1080/10408398.2018.1474170.

[8] Y.M. Ma, L.C. Yuan, B. Wu, X.E. Li, S.L. Chen, S.F. Lu, Genome-wide identification and characterization of novel genes involved in terpenoid biosynthesis inSalvia miltiorrhiza, J. Exp. Bot. 63 (2012) 2809-2823.

[9] X.L. Hao, M. Shi, L.J. Cui, C. Xu, Y.J. Zhang, G.Y. Kai, Effects of methyl jasmonate and salicylic acid on tanshinone production and biosynthetic gene expression in transgenic Salvia miltiorrhiza hairy roots, Biotechnol. Appl. Biochem. 62 (2015) 24-31.

[10] S.J. Zhao, J.J. Zhang, R.H. Tan, L. Yang, X.Y. Zheng, Enhancing diterpenoid concentration in Salvia miltiorrhiza hairy roots through pathway engineering with maize C1 transcription factor, J. Exp. Bot. 66 (2015) 7211-7226.

[11] Q. Huang, M.H. Sun, T.P. Yuan, Y. Wang, M. Shi, S.J. Lu, B.P. Tang, J.X. Pan, Y. Wang, G.Y. Kai, The AP2/ERF transcription factor SmERF1L1 regulates the biosynthesis of tanshinones and phenolic acids in Salvia miltiorrhiza, Food Chem. 274 (2019) 368-375.

[12] X.M. Yan, L. Zhang, J. Wang, P. Liao, Y. Zhang, R. Zhang, G.Y. Kai, Molecular characterization and expression of 1-deoxy-d-xylulose 5-phosphate reductoisomerase (DXR) gene from Salvia miltiorrhiza, Acta Physiol. Plant. 31 (2009) 1015-1022.

[13] W. Gao, M.L. Hillwig, L.Q. Huang, G.H. Cui, X.Y. Wang, J.Q. Kong, B. Yang, R.J. Peters, A functional genomics approach to tanshinone biosynthesis provides stereochemical insights, Org. Lett. 11 (2009) 5170-5173.

[14] J. Guo, Y.J.J. Zhou, M.L. Hillwig, Y. Shen, L. Yang, Y.J. Wang, X.N. Zhang, W.J. Liu, R.J. Peters, X.Y. Chen, Z.B.K. Zhao, L.Q. Huang, CYP76AH1 catalyzes turnover of miltiradiene in tanshinones biosynthesis and enables heterologous production of ferruginol in yeasts, Proc. Natl. Acad. Sci. U. S. A. 110 (2013) 12108-12113.

[15] M. Shi, W. Zhou, J. Zhang, S. Huang, H. Wang, G.Y. Kai, Methyl jasmonate induction of tanshinone biosynthesis in Salvia miltiorrhiza hairy roots is mediated by JASMONATE ZIM-DOMAIN repressor proteins, Sci. Rep. 6 (2016) 20919.
[16] Y. Zhang, E. Butelli, S. Alseekh, T. Tohge, G. Rallapalli, J. Luo, P.G. Kawar, L. Hill, A. Santino, A.R. Fernie, C. Martina, Multi-level engineering facilitates the production of phenylpropanoid compounds in tomato, Nature Comm. 6 (2015) 8635.

[17] H.B. Xu, J.Y. Song, H.G. Luo, Y.J. Zhang, Q.S. Li, Y.J. Zhu, J. Xu, Y. Li, C. Song, B. Wang, W. Sun, G.A. Shen, X. Zhang, J. Qian, A. Ji, Z.C. Xu, X. Luo, L. He, C.Y. Li, C. Sun, H.X. Yan, G.H. Cui, X.W. Li, X.E. Li, J.H. Wei, J.Y. Liu, Y.T. Wang, A. Hayward, D. Nelson, Z.M. Ning, R.J. Peters, X.Q. Qi, S.L. Chen, Analysis of the genome sequence of the medicinal plant Salvia miltiorrhiza, Mol. Plant 9 (2016) 949-952.

[18] G. Zhang, Y. Tian, J. Zhang, L. Shu, S. Yang, W. Wang, J. Sheng, Y. Dong, W. Chen, Hybrid de novo genome assembly of the Chinese herbal plant danshen (Salvia miltiorrhiza Bunge), Gigascience 4 (2015) 62.

[19] W. Zhou, Q. Huang, X. Wu, Z.W. Zhou, M.Q. Ding, M. Shi, F.F. Huang, S. Li, G.Y. Kai, Comprehensive transcriptome profiling of Salvia miltiorrhiza for discovery of genes associated with the biosynthesis of tanshinones and phenolic acids, Sci. Rep. 7 (2017) 10554.

[20] H.Z. Yu, W.L. Guo, D.F. Yang, Z.N. Hou, Z.S. Liang, Transcriptional profiles of SmWRKY family genes and their putative roles in the biosynthesis of tanshinone and phenolic acids in Salvia miltiorrhiza, Int. J. Mol. Sci. 19 (2018) 1593.

[21] M.H. Sun, M. Shi, Y. Wang, Q. Huang, T.P. Yuan, Q. Wang, C. Wang, W. Zhou, G.Y. Kai, The AP2/ERF transcription factor SmERF115 positively regulates the biosynthesis of phenolic acids in Salvia miltiorrhiza, J. Exp. Bot. 70 (2018) 243-254.

[22] J.G. Zhang, L.B. Zhou, X.Y. Zheng, J.J. Zhang, L. Yang, R.H. Tan, S.J. Zhao, Overexpression of SmMYB9b enhances tanshinone concentration in Salvia miltiorrhiza hairy roots, Plant Cell Rep. 36 (2017) 1297-1309.

[23] P.J. Rushton, I.E. Somssich, P. Ringler, Q.J. Shen, WRKY transcription factors, Trends Plant Sci. 15 (2010) 247-258.

[24] T. Eulgem, P.J. Rushton, S. Robatzek, I.E. Somssich, The WRKY superfamily of plant transcription factors, Trends Plant Sci. 5 (2000) 199-206.

[25] J.B. Li, Y.S. Luan, Z. Liu, Overexpression of SpWRKY1 promotes resistance to Phytophthora nicotianae and tolerance to salt and drought stress in transgenic tobacco, Physiol. Plant. 155 (2015) 248-266.

[26] Y.H. Xu, J.W. Wang, S. Wang, J.Y. Wang, X.Y. Chen, Characterization of GaWRKY1, a cotton transcription factor that regulates the sesquiterpene synthase gene (+)- $\delta$ cadinene synthase-A, Plant Physiol. 135 (2004) 507-515.

[27] X. Liu, Y. Song, F. Xing, N. Wang, F. Wen, C. Zhu, GhWRKY25, a group I WRKY gene from cotton, confers differential tolerance to abiotic and biotic stresses in transgenic Nicotiana benthamiana, Protoplasma. 253 (2016) 1265-1281.

[28] U.J. Phukan, G.S. Jeena, R.K. Shukla, WRKY transcription factors: molecular regulation and stress responses in plants, Front. Plant Sci. 7 (2016) 760.

[29] M.H. Chen, T.X. Yan, Q. Shen, X. Lu, Q.F. Pan, Y.R. Huang, Y.L. Tang, X.Q. Fu, M. Liu, W.M. Jiang, Z.Y. Lv, P. Shi, Y.N. Ma, X.L. Hao, L.D. Zhang, L. Li, K.X. Tang, GLANDULAR TRICHOME-SPECIFIC WRKY 1 promotes artemisinin biosynthesis in Artemisia annua, New Phytol. 214 (2017) 304-316.

[30] N. Suttipanta, S. Pattanaik, M. Kulshrestha, B. Patra, S.K. Singh, L. Yuan, The transcription factor CrWRKY1 positively regulates the terpenoid indole alkaloid biosynthesis in Catharanthus roseus, Plant Physiol. 157 (2011) 2081-2093.

[31] J. Chen, D.Y. Shi, S.L. Liu, L. Zhong, Tanshinone IIA induces growth inhibition and apoptosis in gastric cancer in vitro and in vivo, Oncol. Rep. 27 (2012) 523-528.

[32] Y. Zhou, W. Sun, J. Chen, H. Tan, Y. Xiao, Q. Li, Q. Ji, S.H. Gao, Li. Chen, S.L. Chen, L. Zhang, W.S. Chen, SmMYC2a and SmMYC2b played similar but irreplaceable roles in regulating the biosynthesis of tanshinones and phenolic acids in Salvia miltiorrhiza, Sci. Rep. 6 (2016) 22852.

[33] Q.Q. Cheng, P. Su, Y.T. Hu, Y.F. He, W. Gao, L.Q. Huang, RNA interference-mediated repression of SmCPS (copalyldiphosphate synthase) expression in hairy roots of Salvia miltiorrhiza causes a decrease of tanshinones and sheds light on the functional role of SmCPS, Biotechnol. Lett. 36 (2014) 363-369.

[34] Q. Liu, Y. Liu, Y. Tang, J. Chen, W. Ding, Overexpression of NtWRKY50 increases resistance to Ralstonia solanacearum and alters salicylic acid and jasmonic acid production in tobacco, Front. Plant Sci. 8 (2017) 1710.

[35] S. Li, P. Zhang, M. Zhang, C. Fu, L. Yu, Functional analysis of a WRKY transcription factor involved in transcriptional activation of the DBAT gene in Taxus chinensis, Plant Biol. 15 (2013) 19-26.

[36] A.K. Singh, S.R. Kumar, V. Dwivedi, A. Rai, S. Pal, A.K. Shasany, D.A. Nagegowda, A WRKY transcription factor from Withania somnifera regulates triterpenoid withanolide accumulation and biotic stress tolerance through modulation of phytosterol and defense pathways, New Phytol. 215 (2017) 1115-1131.

[37] G.Y. Kai, P. Liao, H. Xu, J. Wang, C.C. Zhou, W. Zhou, Y.P. Qi, J.B. Xiao, Y.L. Wang, L. Zhang, Molecular mechanism of elicitor-induced tanshinone accumulation in Salvia miltiorrhiza hairy root cultures, Acta Physiol. Plant. 34 (2012) 1421-1433.

[38] M. Shi, X.Q. Luo, G.H. Ju, L.L. Li, S.X. Huang, T. Zhang, H.Z. Wang, G.Y. Kai, Enhanced diterpene tanshinone accumulation and bioactivity of transgenic Salvia miltiorrhiza hairy roots by pathway engineering, J. Agric. Food Chem. 64 (2016) 2523-2530.

[39] W. Zhou, F.F. Huang, S. Li, Y. Wang, C.C. Zhou, M. Shi, J. Wang, Y.J. Chen, Y. Wang, H.Z. Wang, G.Y. Kai, Molecular cloning and characterization of two 1deoxy-D-xylulose-5-phosphate synthase genes involved in tanshinone biosynthesis in Salvia miltiorrhiza, Mol. Breeding. 36 (2016) 124. 